\title{
The Effect of Microstructural and Geometric Inhomogeneities Induced by Laser for Forming Strain Analysis on Sheet Metal Formability
}

\author{
Sergey Guk, Daria Plotnikova, Rudolf Kawalla \\ Institute for Metal Forming, TU Bergakademie Freiberg, Freiberg, Germany \\ Email: Sergey.Guk@imf.tu-freiberg.de
}

Received 18 April 2016; accepted 17 May 2016; published 20 May 2016

Copyright (C) 2016 by authors and Scientific Research Publishing Inc.

This work is licensed under the Creative Commons Attribution International License (CC BY).

http://creativecommons.org/licenses/by/4.0/

(c) (i) Open Access

\begin{abstract}

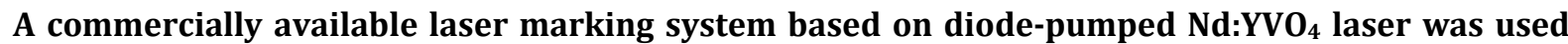
for creating grid patterns for forming strain analysis of a dual-phase steel. The aim was to determine and analyze the influence of laser working parameters on the formability of sheet material by means of an in-depth characterization of this induced microstructural and geometric inhomogeneity. The electrochemical etching served as the reference method without the negative effect of generating inhomogeneity. The formability was evaluated using the cupping test according to Erichsen. While the quantification of geometric inhomogeneity was based on the determination of the notch factor, light microscopy and microhardness measurement were used for the evaluation of microstructural inhomogeneity. Furthermore, on the basis of the results an empirical regression model was established which described in terms of quantity the relationship between the examined factors such as laser power, pulse frequency and scanning speed as well as the command variable and the mark depth. The results showed that microstructural inhomogeneity in the used marking parameters due to their locally very limited formation did not have an appreciable influence on the mechanical properties. In contrast to this, the induced geometric inhomogeneity had a marked influence on the material formability.
\end{abstract}

\section{Keywords}

Laser Grid Marking, Deformation Analysis, Inhomogeneity, Sheet Metal, Formability 


\section{Introduction}

One of the major tools in the optimization of the forming process of a material with regard to component quality and component failure, respectively, process parameters and security is the local deformation analysis. It finds a particularly wide application in the field of sheet metal forming. For a local deformation analysis, various methods are available. The conventional method is grid measurement. To measure the local strain, a grid pattern with different shapes (e.g. circular or crossing lines) is plotted on the sheet metal blank surface prior to forming. After completion of the forming process, the distorted grid pattern is measured in order to determine the major and minor strain.

For production of the grid pattern on the sheet surface, various methods are known [1]. Among the most common methods are electrochemical etching, screen printing and laser marking. They differ in terms of accuracy, contrast, durability, quality and cost. Especially laser grid patterning, as a relatively new process and due to its currently reduced investment costs, rapidness and accuracy, has considerably gained in applications in the laboratory as well as under industrial conditions.

Nevertheless, a quick application of laser marking requires an optimization of marking parameters in terms of a good visual recognizability of the grid pattern by a computerized strain measurement system. In most cases, the effect of the marking is produced by means of color change accompanied by a surface structuring. Depending on laser power, pulse frequency and scanning speed, it is possible to either warm, melt or vaporize the material. The crucial task is to optimize the patterning parameters in order to prevent the negative influence of the grid on the changing or weakening of local material properties. These local changes can be induced by microstructural [2] and/or geometric inhomogeneities [3] in the vicinity of the grid, especially for multiphase steels such as DP- or TRIP-steels, and lead to poorer formability of the material [4]. While the microstructural inhomogeneity is associated with local temperature-induced structural changes (e.g. tempering), the geometric inhomogeneity represents a deviation from the regular geometry on the sample length. This non-uniformity of microstructure and geometry may produce, depending on their size, a non-uniform distribution of tension and consequently an uneven distribution of strain over the sample length. As part of the investigations described a commercially available laser marking system for a dual-phase steel grid patterning was used. The influence of the marking parameters on the two types of inhomogeneity was extensively investigated by experimentation. By means of an established empirical model, the influence of the marking parameters on the geometrical inhomogeneity was generalized. For evaluation of the effect of laser marking parameters on the material formability, an Erichsen test was conducted. Thereby the electrochemical etching served as the reference method for production of the grid pattern without the negative effect of generating inhomogeneity. In this way a complete description of the patterning parameters effects during laser marking was accomplished in order to facilitate the final, optimal selection that was characterized by a low geometric and microstructural inhomogeneity combined with a good visual recognizability of the grid pattern.

\section{State of the Art}

For a local deformation analysis different methods are available, with the grid measurement method playing a central role. For producing a grid pattern on the material surface methods such as electrochemical etching, screen printing or laser marking are suitable. Among these, electrochemical etching is one of the most commonly used methods for sheet metal marking [5]. In this method the pattern design of a plastic template is etched on the sheet material by means of an alternating current mark. Placed on top of the template is a cover of carbonaceous felt mat that is impregnated with an electrolyte solution. A steel roller rolls across the felt which is, as well as the metal blank, switched on in the AC circuit. The carbon in the felt delivers dark deposit products (oxides) which attach to all places on the sheet surface that have electrical contact. In this way a black measurement grid on the metal blank is formed without corrosion or other adverse interference that occurs in the material's appearance [6]. The main advantages of this method are its simplicity and its low technical costs. The resulting pattern lines feature a good abrasion resistance and cause no stress concentrations during forming out. Furthermore, accurate measurement grid patterns can be obtained at which there is no risk of local microstructure changes due to low process temperature. The main disadvantages of the method are the applicability only on electrically conductive surfaces as well as difficulties when there is a faster changeover of parameters of the grid lines, because in this case a new template is required [7].

Screen printing is one of the oldest measurement grid methods. The method was discovered in China almost 
2.000 years ago. In this method the measurement grid is applied directly to the metal blank using a suitable ink. The main advantages of screen printing are simplicity, ease of use, low cost and availability of resources in terms of materials, labor and technical knowledge. In addition, the grid color can be freely selected, for example to increase the contrast. However, the measurement grid has a low abrasion resistance and chipping may occur in the course of forming when the surface area is strongly enlarged.

The laser marking process is a relatively new method for applying a measurement grid. During this process an induced laser beam produces a mark on the material surface by one or more mechanisms such as engraving, material removing, tempering, discoloration or foaming. In engraving the power density of the laser beam is so high that the material partially melts and vaporizes. Thereby, cavities are formed on the material surface. Mäntyjärvi et al. verified that oxides are formed on the brick of the cavities and their color makes the grid marking visible [8]. This method can be used for metals, ceramics and some plastics [9]. During material removal the top layers that are spread over the test material are removed by a laser beam. The contrast in this case is produced by color differences between the two layers. Frequently used materials that are laser-marked by material removal are anodized aluminum, varnished metals, films or laminates. The tempering marking method can be applied for certain metals. It makes use of the characteristic behavior of metals to change their color during heating and subsequent cooling. It is important to note that with tempering marking no surface damage occurs. Applications of this method are reported for example for surgical instruments in [10]. A specific type of the laser marking process is discoloration and foaming, used exclusively for plastics. Therefore, in this context, they are not elucidated further, as clarified in [9].

Out of the whole range of existing laser systems on the market such as the $\mathrm{CO}_{2}$, fiber and crystal lasers, only the latter has been able to prevail for the processing of metal and its grid marking. So an Nd:YAG laser has a tenfold shorter wavelength compared to a $\mathrm{CO}_{2}$ laser, which enables it to be focused to smaller areas. According to [9] the grid marking is produced either by the vector or by the scanning method. In both methods the obtained surface roughness and the mark depth of the laser beam respectively play an important role for the determination of contrast and a good visual recognizability of the grid marking. Leone et al. state that the relationship between the contrast and the roughness $R_{t}$ is displayed by means of a root function [11]. The influence of occurred oxidation in the form of oxygen content on the visual contrast can be described by linear dependence [11]. In several studies the predominant influence of the laser power, pulse frequency and scanning speed on the grid marking geometry [11]-[13] and quality [9] [14] was demonstrated. Furthermore, Abeln gives an account of a stronger interaction of the laser beam with the material as the laser power increases, which becomes evident on the material surface by a measured roughness, mark depth or rather an erosion rate [15]. The influence of the laser pulse frequency on the mark depth and width has been investigated in [11] [16]. It was found that there is almost no dependence of mark width on the pulse frequency. In contrast to this, mark depth increases up to a power related point of inflection as pulse frequency is increased; at this point the continuous mark depth increase is switched to a monotonous decrease. The authors explain this phenomenon by an interaction between the laser power and the pulse frequency in relation to the incoming material evaporation in the focal point. Furthermore, the oxidation can be activated by increasing the pulse frequency [16]. The impact of the grid marking quality by varying scanning speeds was investigated by Mäntyjärvi et al. [8]. From this it follows that the effect of scanning speed depends strongly on its relationship to pulse frequency. The limiting states here are far apart lying or rather strongly overlapping laser points.

\section{Materials and Experimental Arrangement}

The studied material was a cold rolled dual-phase steel HCT600X + Z140 sheets $1 \mathrm{~mm}$ in thickness. The steel was hot-dip galvanized with the respective zinc coating thickness of 10 microns per side. The initial microstructure of the material can be seen in Figure 1. It primarily consists of ferritic matrix (brown) and of martensite (white) at the grain boundaries.

The square grid measurement $1 \times 1 \mathrm{~mm}^{2}$ with the line width of $0.15 \mathrm{~mm}$ was applied on the examined material surface both electrochemically by means of the Electrolytic marking EU Classic System (reference method) and by means of the laser marking system Lasebox XS of the company Östling Marking Systems GmbH. The laser marking system is based on the use of a diode-pumped $\mathrm{Nd}: \mathrm{YVO}_{4}$ laser source having a wavelength of 1.064 microns and a beam diameter of $6 \mathrm{~mm}$. The focal point diameter is approximately 50 microns in the focus. The marking system allows the following variation of the marking parameters: laser power from 5 to $20 \mathrm{~W}$, 


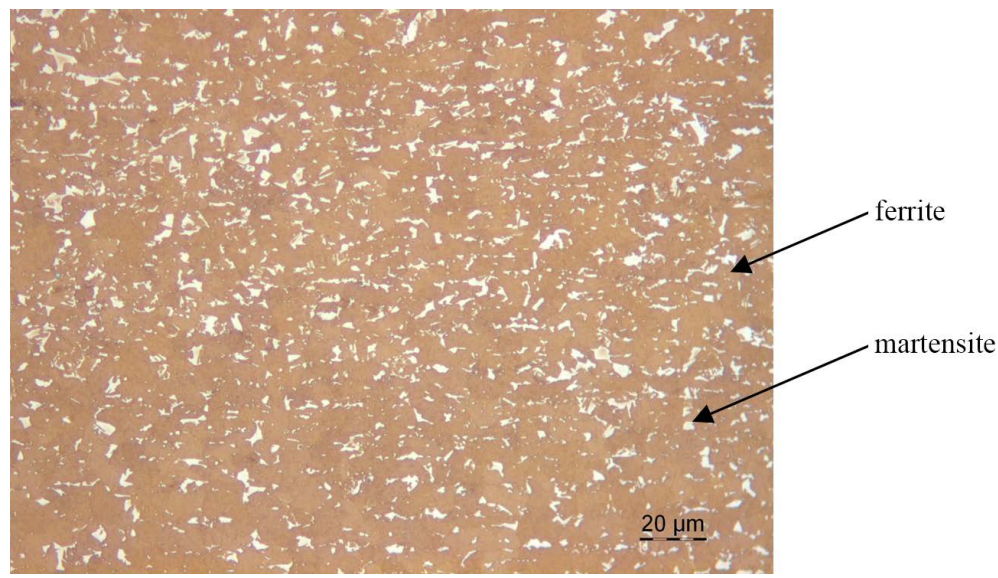

Figure 1. Microstructure of steel HCT600X + Z140; Le Pera etched.

pulse frequency between 10 and $150 \mathrm{kHz}$ and scanning speed from 10 to $5000 \mathrm{~mm} / \mathrm{s}$. The wider track of 0.15 $\mathrm{mm}$ was made using two tracks next to each other. The material temperature measurement in the direct vicinity of the focal point at the time of contact between laser beam and metal surface was carried out using a type $\mathrm{K}$ thermocouple.

The full range of marking parameters was checked first with regard to good visual grid recognizability using an available deformation measurement system AutoGrid ${ }^{\circledR}$ from the company ViALUX. For a group of the optimal marking parameters selected in this way followed a subsequent mark depth measurement and the determination of steel microstructure change in the course of laser beam thermal action at $500 \times$ magnification by light microscopy. As an etching agent for color representation Le Pera was used. For the characterization of the mechanical properties in the laser treatment zone, microhardness measurements HV0.1 were performed according to ISO 6705-1 at the hardness tester ZHV1. The microhardness measurement was effected with gradually increasing depth both under the laser line and below the edge without laser grid marking. Special consideration was given to the condition that the distance between the respective measuring point and the sheet surface in both cases was the same. The formability of grid marked sheet blanks with optimal marking parameters was assessed on the basis of the Erichsen cupping test in accordance with DIN EN ISO 20482. The tests were performed on the sheet metal forming testing machine BUP600 with a spherical punch head of diameter $20 \mathrm{~mm}$. The formability was evaluated by means of the height of the cup at the moment of sheet metal fracture, defined as the Erichsen cupping value IE.

\section{Experimental Results}

The assessment of the marking parameter with respect to the good grid marking visual recognizability by the use of the deformation measuring system AutoGrid ${ }^{\circledR}$ allowed to set limits regarding the optimal parameters for the investigated material. The following are the optimal parameters: laser power from 16 to $20 \mathrm{~W}$, pulse frequency between 40 and $80 \mathrm{kHz}$ as well as scanning speed from 10 to $50 \mathrm{~mm} / \mathrm{s}$. Thereby the material maximum measured temperature in the direct vicinity of the focal point at the time of contact between laser beam and metal surface was $350^{\circ} \mathrm{C}$. This temperature showed no significant change in the optimum range of the laser parameters. The measured mark depth results are shown in Figure 2 and Figure 3. The determination of the mark depth was carried out by means of the gray value correlation technique and showed a standard deviation of less than $10 \%$. It can be seen that with an increase of the laser power or a decrease of the scanning speed there is a nonlinear growth of the mark depth. It is to note that the visual grid recognizability using the system AutoGrid ${ }^{\circledR}$ does not work when the mark depth is in the range of the zinc layer thickness.

The microstructural evolution in the course of laser beam thermal action is exemplified in Figure 4. It can be seen that there is a visually recognizable grid line which is formed for technological reasons from two closely spaced adjacent tracks. The results show that a local phase transformation of the martensite into bainite takes place within a range of about 40 microns away from the track edge in a wide range of pulse frequencies at a laser power of $18-20 \mathrm{~W}$ and a scanning speed of less than $30 \mathrm{~mm} / \mathrm{s}$ (Figure 4(a), Figure 4(b)). A reduction of 


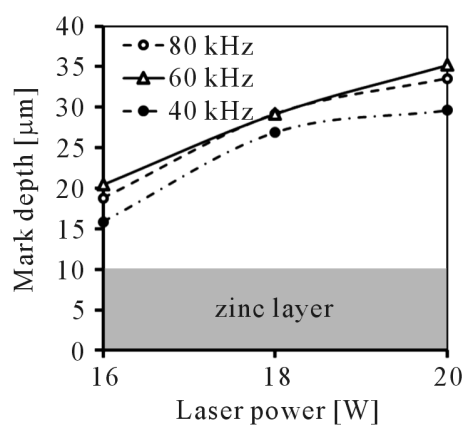

(a)

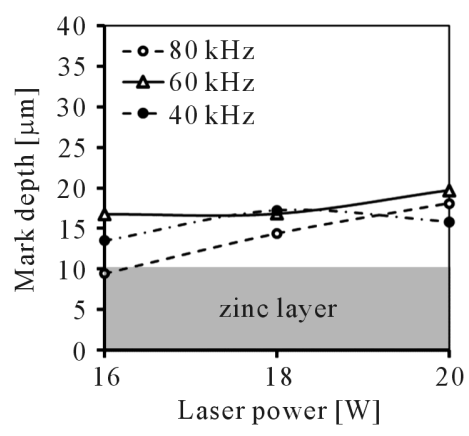

(b)

Figure 2. Mark depth against laser power for different values of pulse frequency. Scanning speed of $10 \mathrm{~mm} / \mathrm{s}$ (a) and $30 \mathrm{~mm} / \mathrm{s}$ (b).

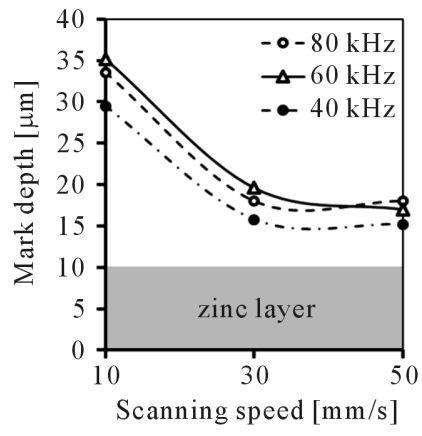

(a)

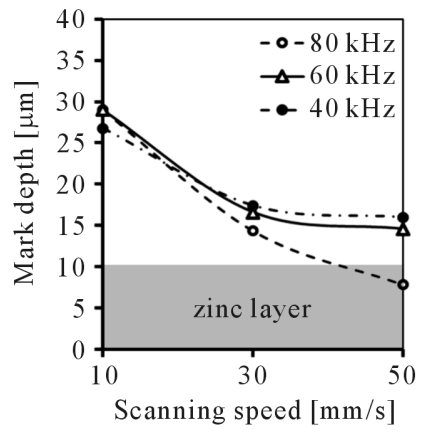

(b)

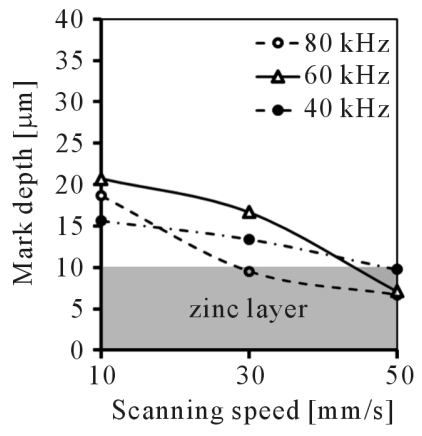

(c)

Figure 3. Mark depth against scanning speed for different values of pulse frequency. Laser power of $20 \mathrm{~W}$ (a) $18 \mathrm{~W}$ (b) and $16 \mathrm{~W}$ (c).

laser power and an increase of scanning speed lead to decreased depth of local microstructural changes of about 15 microns away from the track edge (Figure 4(c)).

On samples that showed a significant microstructural change the microhardness measurement HV0.1 was performed. The results are similar for all selected combinations of parameters within the permitted tolerances. Figure 5 shows that no changes of mechanical properties were detected in the direct vicinity of the tracks.

Finally, Table 1 shows the Erichsen cupping test results at the grid marked sheet blanks with optimal laser marking parameters. It can be seen that for the mark depth less than 17 microns the reached Erichsen cupping value IE increases from $9.1 \mathrm{~mm}$ to $9.7 \mathrm{~mm}$. The further decrease of the mark depth leads to no changes of IE value. These values correspond with those of the electrochemically grid marked metal blanks (IE $=9.8 \mathrm{~mm})$. An additional visual indication to be mentioned is that for the mark depth above 17 microns the cracks proceeded along the lasered grid. It implies that the grid has changed or weakened the material properties locally. If the mark depth is less than 10 microns there is only a partial removal of the zinc layer and consequently an insufficient visual recognizability of the grid marking.

\section{Discussion}

As the results of the studies show, a local microstructural inhomogeneity in the vicinity of the grid at $18-20 \mathrm{~W}$ laser power and a scanning speed below $30 \mathrm{~mm} / \mathrm{s}$ occurs. Nevertheless, it is found in a distance of approximately 40 microns from the track edge and leads to no significant influence on the material mechanical properties. The measured HV0.1 values are in good agreement with work of Krajewski et al. [17]. In this work the average microhardness of ferrite in this type of steel was $220 \mathrm{HV} 0.1$ and of martensite $384 \mathrm{HV} 0.1$. In addition to the insignificant microstructural inhomogeneity, the laser grid marking leads to the formation of a geometric inhomogeneity. It occurs on the material surface in the form of notches. In order to evaluate the geometrical inhomogeneity, the established notch stress and the notch factor of the flat notches according to Bänki is used [18]. The notch factor can be calculated with the following approach. 


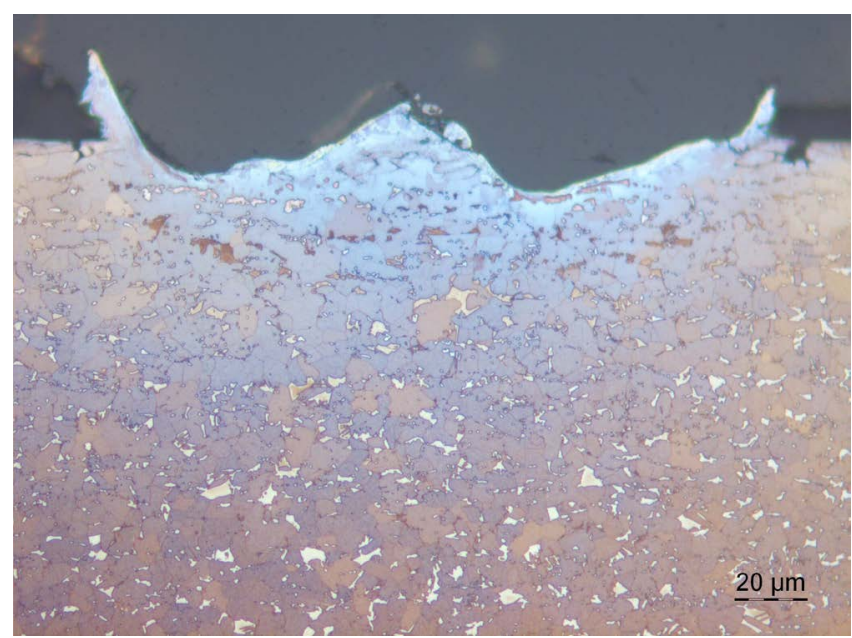

(a)

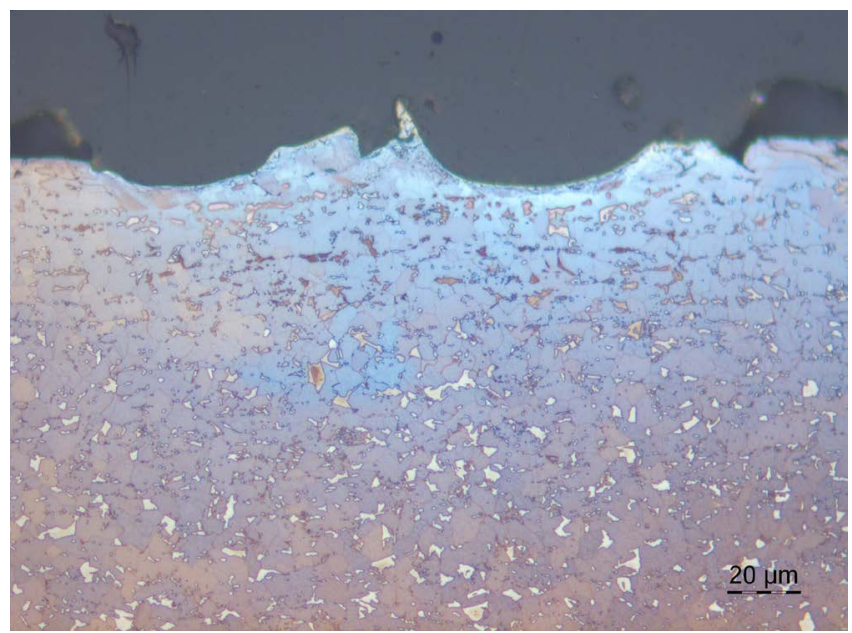

(b)

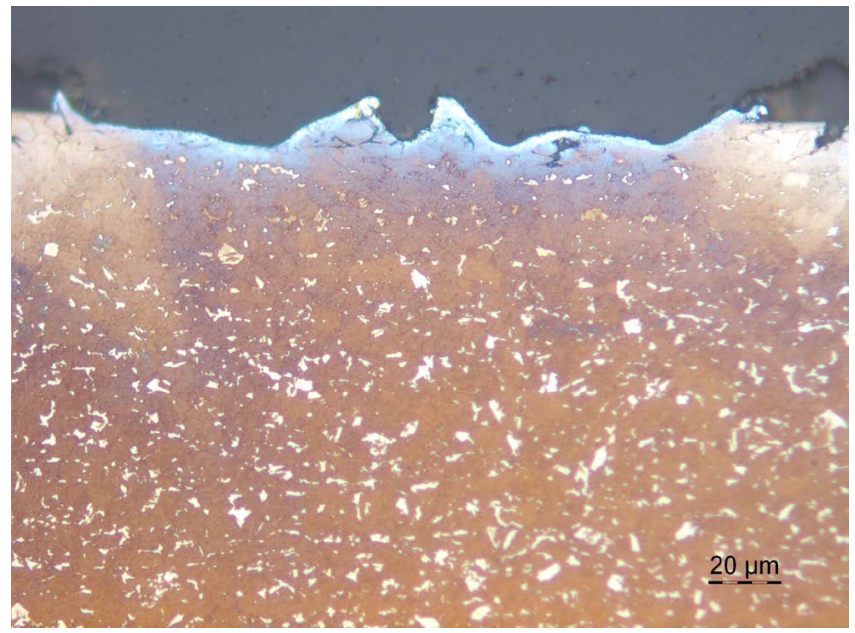

(c)

Figure 4. Etched crosscut of laser grid. Bainite-dark brown/ black, ferrite - blue/brown, martensite - white. Pulse frequency of $60 \mathrm{kHz}$, scanning speed of $25 \mathrm{~mm} / \mathrm{s} \mathrm{(a),} \mathrm{(b)} \mathrm{and} 50 \mathrm{~mm} / \mathrm{s}$ (c), laser power of $20 \mathrm{~W}$ (a), $18 \mathrm{~W}$ (b) and $16 \mathrm{~W}$ (c). 


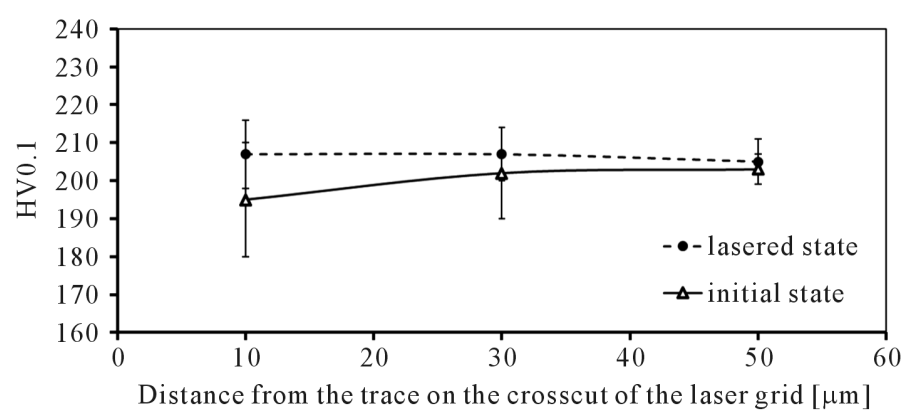

Figure 5. Microhardness measurements in the direct vicinity of the tracks. Laser power of $20 \mathrm{~W}$, pulse frequency of $60 \mathrm{kHz}$ and scanning speed of $25 \mathrm{~mm} / \mathrm{s}$.

Table 1. Erichsen cupping values for various laser marking parameters.

\begin{tabular}{ccccc}
\hline \multirow{2}{*}{ Mark depth, $\mu \mathrm{m}$} & \multicolumn{4}{c}{ Variable laser parameters } \\
\cline { 2 - 4 } & $\begin{array}{c}\text { Laser power, } \\
\text { W }\end{array}$ & $\begin{array}{c}\text { Pulse frequency, } \\
\text { kHz }\end{array}$ & $\begin{array}{c}\text { Scanning speed, } \\
\mathrm{mm} / \mathrm{s}\end{array}$ & $\begin{array}{c}\text { Erichsen cupping } \\
\text { value IE, mm }\end{array}$ \\
19 & 18 & 60 & 25 & 9.1 \\
18 & 20 & 80 & 30 & 9.4 \\
16 & 18 & 60 & 30 & 9.7 \\
15 & 18 & 40 & 50 & 9.7 \\
14 & 20 & 40 & 50 & 9.7 \\
13 & 18 & 80 & 30 & 9.7 \\
12 & 16 & 40 & 30 & 9.8 \\
11 & 16 & 60 & 40 & 9.8 \\
10 & 16 & 60 & 40 & 9.9 \\
9 & 16 & 40 & 50 & unrecognizable \\
8 & 16 & 80 & 30 & unrecognizable \\
7 & 16 & 80 & 40 & unrecognizable \\
\hline
\end{tabular}

$$
n=1+2 \sqrt{t / \rho},
$$

where $\rho=\left(a^{2}+4 t^{2}\right) / 8 t, t$ is the mark depth; $a$ is the mark width and $\rho$ is the radius of curvature. By measuring the tracks on grid marking with various marking parameters a correlation of the calculated notch factor with the Erichsen cupping value can be established (Figure 6). It can be seen that a significant reduction in the material formability occurs with a notch factor above 1.65. The results can be used for selecting those marking parameters that lead to different track geometries in order to prevent geometrical inhomogeneity that reduces material formability.

Based on the presented results for the mark depth, the marking parameters were conclusively assessed according to their weighting. This was done by establishing an empirical model that quantitatively describes the relationship between the examined factors such as laser power, pulse frequency and scanning speed as well as the command variable as mark depth. For this purpose, the method of central multi-part full factorial design was applied as described by Hofmann [19]. As already reported by Leone et al. [11], a non-linear functional analysis was assumed. For this reason, the polynomial coefficients were determined from an orthogonal central multipart design 2 nd order. To check the significance of the regression coefficients, the t-test with the significance 
level of $95 \%$ was performed according to [20]. Based on the boundary conditions, the following relationship was established:

$$
t=-43.41+0.676 L+0.51 F+0.022 G-0.007 L \cdot G-0.0043 F^{2}+0.00582 G^{2}
$$

where $t$ is the mark depth, $L$ is the laser power, $F$ is the pulse frequency and $G$ is the scanning speed. As the relationship shows, the laser power and the pulse frequency have a dominant influence on the mark depth. The visualization of the regression equation in the form of impact surfaces can be seen in Figure 7. The selected color scale with the red color indicates the area of the marking parameters which presents a material formability influence. The accuracy assessment of the established model revealed that only for 3 from 27 calculated marking parameter combinations the mark depth deviation of the measured values exceeded 5 microns.

Furthermore, it follows from Figure 7 that the pulse frequency of $60 \mathrm{kHz}$ represents an inflection point that is independent of laser power and scanning speed and at which a continuous mark depth increase is switched to a monotonous decrease.

\section{Conclusion}

As the investigations have shown, in the field of grid measurement technique the laser grid marking for applying a grid measurement on the material surface can be successfully used for performing a local deformation analysis. This was confirmed on the basis of the present laser marking system Lasebox XS with the maximum laser power of $20 \mathrm{~W}$ at the dual-phase steel HCT600X + Z140. However, in the selection of marking parameters attention must be

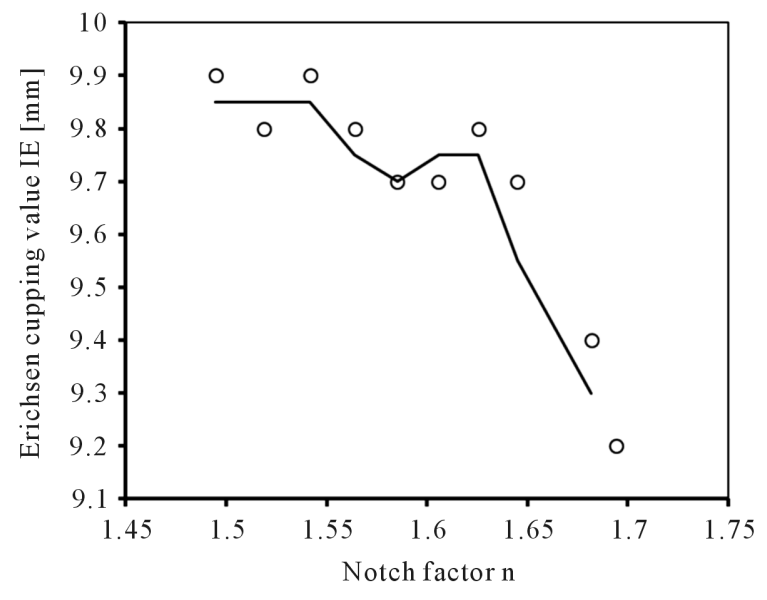

Figure 6. Erichsen cupping value against notch factor.
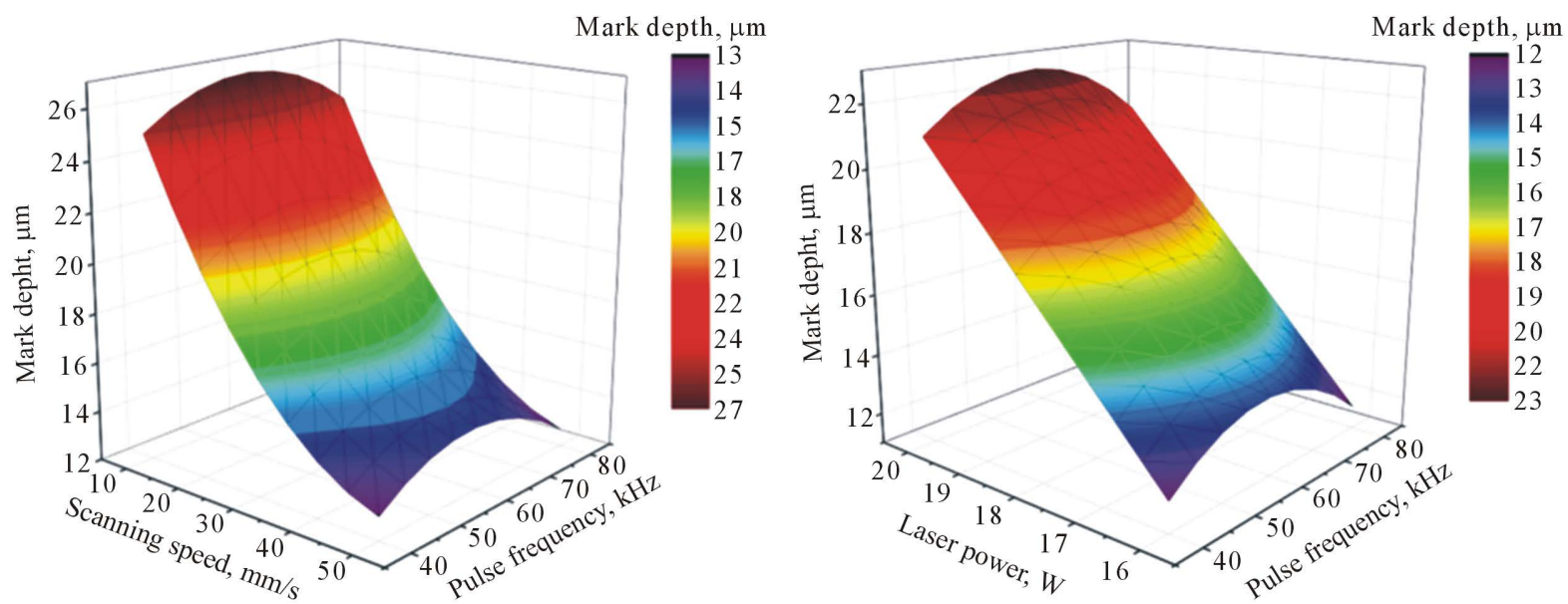

Figure 7. Impact area of the mark depth against scanning speed and pulse frequency for laser power of $18 \mathrm{~W}$ (a) as well as against laser power and pulse frequency for scanning speed of $30 \mathrm{~mm} / \mathrm{s}(\mathrm{b})$. 
paid, besides a good visual recognizability of a grid, to the microstructural and geometric inhomogeneities. While microstructural inhomogeneity with the used marking parameters due to their extremely local formation didn't appreciably influence the mechanical properties, the induced geometric inhomogeneity had a marked influence on material formability. This was determined using the Erichsen cupping test. Thereby the notch factor calculation was used for the quantification of the geometric inhomogeneity. Furthermore, based on the results an empirical regression model was established, which quantitatively described the relationship between the examined factors such as laser power, pulse frequency and scanning speed as well as the command variable as mark depth. In summary, it can be stated that these two methods provide a useful input to the laser mark depth determination as a function of marking parameters and ultimately contribute to the selection of optimal values with regard to the visual recognizability as well as the induced geometric inhomogeneity during the application of a measurement grid on the material surface.

\section{References}

[1] Guk, S. (2006) The Relationship between Microstructure and Material Flow Processes in Sheet Metal Forming (German). PhD Thesis, TU Bergakademie Freiberg, Freiberg.

[2] Thenikl, T., Guk, S. and Vallen, H. (2011) Interaction between Material Flow and Microstructure Evolution with Acoustic Emission Measurement. Proceedings of the International Conference MEFORM, Freiberg, 30 March-1 April 2011, 397-404. http://www.gbv.de/dms/tib-ub-hannover/657990396.pdf

[3] Guk, S., Smirnov, O. and Kawalla, R. (2006) Nonuniformity of the Deformation of Microstructural Components of Multiphase Steels in Evaluating the Limiting Formability of Flat-Rolled Products. Metallurgist, 5, 219-224. http://link.springer.com/article/10.1007\%2Fs11015-006-0067-9

[4] Guk, S. (2016) Evaluation of Formability of Thin Sheet Metal from Mechanical Properties. Key Engineering Materials, 684, 80-85. http://dx.doi.org/10.4028/www.scientific.net/kem.684.80

[5] Ozturk, F., Dilmec, M., Turkoz, M., Ece, R. and Halkaci, H. (2009) Grid Marking and Measurement Methods for Sheet Metal Formability. Proceedings of the 5th International Conference and Exhibition on Design and Production of Machines and Dies/Molds, Kuşadas1, 18-21 June 2009, 41-49.

[6] Bleck, W. (1999) Material Testing in the Study and Practice (German). Günter Mainz, Mainz.

[7] Schatz, M. (2010) Advanced Applications of the Method of the Visio Plasticity in Sheet Metal Forming (German). $\mathrm{PhD}$ Thesis, TU Dresden, Dresden.

[8] Mäntyjärvi, K., Tulonen, J., Saarnivuo, T., Porter, J. and Karjalainen, J.A. (2008) Grid Patterns by Forming Strain Analysis. International Journal of Material Forming, 4, 249-252. http://dx.doi.org/10.1007/s12289-008-0355-y

[9] Technical Information for Laser Marking (German) (2006) TRUMPF Laser Marking Systems AG. http://www.trumpf-laser.com/fileadmin/DAM/trumpf-laser.com/Technische Infos/TI Laserbeschriften.PDF

[10] Laser Magazine of Trumpf (German)(2013) Laser-Community. http://www.laser-community.com/de/metall-mit-laser-markieren-reicht-von-beschriften-ueber-gravieren-oder-abtragen -bis-zu-anlassen/

[11] Leone, C., Genna, S., Caprino, G. and Iorio, I. (2010) AISI 304 Stainless Steel Marking by a Q-Switched Diode Pumped Nd:YAG Laser. Journal of Materials Processing Technology, 10, 1297-1303. http://dx.doi.org/10.1016/j.jmatprotec.2010.03.018

[12] Lallemand, G., Jacrot, G., Cicala, E. and Grevey, D.F. (2000) Grooving by Nd:YAG Laser Treatment. Journal of Materials Processing Technology, 1-3, 32-37. http://dx.doi.org/10.1016/S0924-0136(99)00256-3

[13] Cicala, E., Soveja, A., Sallamand, P., Grevey, D. and Jouvard, J.M. (2008) The Application of the Random Balance Method in Laser Machining of Metals. Journal of Materials Processing Technology, 1-3, 393-401. http://dx.doi.org/10.1016/j.jmatprotec.2007.05.049

[14] Hügel, H. and Graf, T. (2009) Lasers in Manufacturing. Laser Sources, Systems, Manufacturing Processes (German).

[15] Abeln, T. (2002) Fundamentals and Process Engineering of Reactive Laser Precision Ablation of Steel (German). PhD Thesis, TU Stuttgart, Stuttgart.

[16] Qi, J., Wang, K. and Zhu, Y. (2003) A Study on the Laser Marking Process of Stainless Steel. Journal of Materials Processing Technology, 1-3, 273-276. http://dx.doi.org/10.1016/S0924-0136(03)00234-6

[17] Krajewski, S. and Nowacki, J. (2014) Dual-Phase Steels Microstructure and Properties Consideration Based on Artificial Intelligence Techniques. Archives of Civil and Mechanical Engineering, 2, 278-286. http://dx.doi.org/10.1016/j.acme.2013.10.002

[18] Bänki, I. (1966) Comments on the Notch Factors Specified by Neuber for Thin Flat Panels (German). Mechanical En- 
gineering, 3, 257-269.

[19] Hofmann, M. (2011) Development and Validation of an Evaluation Model for Projects of the Static Design of Experiments (German). PhD Thesis, TU Berlin, Berlin.

[20] Maslov, G. and Zibulevskiji, V. (2007) Optimization of Parameters and Operating Modes of Machines by Means of Design of Experiments (Russian). UMC "Triada", Moscow. 